\title{
Right upper lobe anatomy revisited: a computed tomography scan study
}

\section{L'anatomie du lobe supérieur droit revisitée : une étude par tomodensitométrie}

\author{
Jean S. Bussières, MD, FRCPC (D) • Michel Gingras, MD, FRCPC • Lindsay Perron, MD • \\ Jacques Somma, MD, BEng, FRCPC • Marili Frenette, MD • Etienne J. Couture, MD, FRCPC • \\ Olivier Moreault, MD · Yves Lacasse, MD, MSc, FRCPC
}

Received: 23 April 2018/Revised: 31 October 2018/Accepted: 26 February 2019/Published online: 5 March 2019

(C) Canadian Anesthesiologists' Society 2019

\begin{abstract}
Background The double lumen tube (DLT) has become the most popular way to isolate the lungs for thoracic surgery. The variable anatomy of the right main stem bronchus (RMSB) seems to be the main reason clinicians are unwilling to use the right-sided DLT (R-DLT). The factors that could compromise the adequate ventilation of the right lung are mostly the variable length of the RMSB and the misalignment of the lateral orifice of the R-DLT in regard to the right upper lobe bronchus (RULB). The objectives of this study were to validate an alternative method to estimate the RMSB length, and to determine the distribution of the angulation of the ostium of the RULB. Methods From high-resolution computed tomography scans of the thorax of 106 consecutive patients, the length of the RMSB was measured using Kim's method and the carina-to-carina method. The angle between the $R U L B$ origin and the lateral aspect of the RMSB was also
\end{abstract}

J. S. Bussières, MD, FRCPC ( $\square)$

Department of Anesthesiology, Institut universitaire de cardiologie et de pneumologie de Québec - Université Laval, 2725, Chemin Sainte-Foy, Quebec City, QC G1V 4G5, Canada e-mail: jbuss@criucpq.ulaval.ca

Department of Anesthesiology and Critical Care, Université Laval, Quebec City, QC, Canada

M. Gingras, MD, FRCPC

Department of Radiology, Institut universitaire de cardiologie et de pneumologie de Québec - Université Laval, Quebec City, QC, Canada

L. Perron, MD

Department of Radiology, Université Laval, Quebec City, QC, Canada measured. All these measurements were correlated and inter-observer variation documented.

Results From the Kim's method, the mean (standard deviation [SD]) length of the RMSB was 25.5 (4.7) $\mathrm{mm}$. From the alternative carina-to-carina method, the mean (SD) length of RMSB was 29.4 (4.6) $\mathrm{mm}$. The interobserver agreement was substantial with both methods (Kim's method: intraclass correlation coefficient [ICC] = 0.84; carina-to-carina method: ICC $=0.95)$. Both measures were closely related $(I C C=0.93 ; P<0.001)$. The RULB presented a wide range angulation [mean $(S D)$, $0.1(9.5)^{\circ}$; range, -28.6 to 21.2$]$.

Conclusion These anatomic observations provide a better understanding of the variable anatomy of the right bronchial tree and may guide thoracic anesthesiologists in the choice of the best lung isolation device for their patients.

\section{J. Somma, MD, BEng, FRCPC}

Department of Anesthesiology, Institut universitaire de cardiologie et de pneumologie de Québec - Université Laval, 2725, Chemin Sainte-Foy, Quebec City, QC G1V 4G5, Canada

M. Frenette, MD · E. J. Couture, MD, FRCPC · O. Moreault, MD Department of Anesthesiology and Critical Care, Université Laval, Quebec City, QC, Canada

Y. Lacasse, MD, MSc, FRCPC

Multidisciplinary Department of Respiratory Medicine and Thoracic Surgery, Institut universitaire de cardiologie et de pneumologie de Québec - Université Laval, Quebec City, QC, Canada 


\section{Résumé}

Contexte Le tube double lumière (TDL) est actuellement la modalité la plus populaire pour isoler les poumons lors d'une chirurgie thoracique. L'anatomie variable de la bronche souche droite (BSD) semble être la principale raison qui freine l'engouement des cliniciens à utiliser un $T D L$ droit $(T D L-d)$. Les facteurs pouvant compliquer la ventilation adéquate du poumon droit sont surtout la longueur variable de la BSD et le mauvais alignement entre l'orifice latéral du TDL-d et de la bronche lobaire supérieure droite (BLSD). Les objectifs de cette étude étaient de valider une méthode alternative d'estimation de la longueur de la BSD ainsi que de déterminer la distribution de l'angulation de l'orifice de la BLSD.

Méthode En nous basant sur des images de tomodensitométrie (TDM) thoracique haute résolution de 106 patients consécutifs, la longueur de la BSD a été mesurée à l'aide de la méthode de Kim et de la méthode de carène à carène. L'angle entre l'origine de la BLSD et l'aspect latéral de la BSD a également été mesuré. Toutes ces mesures ont ensuite été corrélées et la variation interobservateur documentée.

Résultats En se fondant sur la méthode de Kim, la longueur moyenne (écart type [ÉT]) de la BSD était de 25,5 (4,7) $\mathrm{mm}$. En se fondant sur la méthode alternative de carène à carène, la longueur moyenne (ÉT) de la BSD était de 29,4 (4,6) $\mathrm{mm}$. La concordance inter-observateur était élevée lors de l'utilisation des deux méthodes (méthode de Kim : coefficient de corrélation intraclasse $[\mathrm{CCI}]=0,84$; méthode carène à carène : 0,95). Les deux mesures étaient très rapprochées $(C C I=0,93 ; P<0,001)$. La BLSD présentait une angulation très variable [(ÉT) moyen, 0.1 $(9,5)^{\circ}$; éventail, $-28,6$ à 21,2$]$.

Conclusion Ces observations anatomiques nous permettent de mieux comprendre l'anatomie variable de l'arbre bronchique droit et pourrait aider les anesthésiologistes thoraciques à choisir le dispositif le mieux adapté pour isoler le poumon de leur patient.

In the early 1980s, the introduction of the disposable double lumen endotracheal tube (DLT), combined with the use of fibreoptic bronchoscopy (FOB) to confirm its positioning, contributed to making the DLT the gold standard for lung isolation in thoracic surgery. Nevertheless, the right-sided DLT (R-DLT) is still less used. The relative variability of the anatomy of the right tracheobronchial tree compared with the left main stem seems to be the principal reason clinicians are unwilling to use the R-DLT. ${ }^{1}$
In 2002, Cohen summarized the status of the R-DLT: "There is an ongoing debate as to whether a right-sided DLT should be used routinely in thoracic surgery. The difficulty in properly positioning the right-sided DLT results not only from the depth of insertion, but also from turning the tube left or right to allow the ventilating port to lie against the orifice of the right upper lobe." 2 Ten years later, "the eternal debate on how to choose the DLT side" was still present in the literature. ${ }^{3}$

The depth of insertion of the R-DLT obviously relates to the length of the right main stem bronchus (RMSB). Kim et al. observed a higher incidence of R-DLT malpositioning and ventilation problems as well as postoperative lung collapse when the length of RMSB was $<23 \mathrm{~mm}$ on a computed tomography (CT) scan (Figs 1A and 2). ${ }^{4}$ The authors concluded that measurement on a thoracic CT scan is useful for clinicians to predict successful utilization of an R-DLT, and suggested that a left-sided tube should be used when the length of the RMSB is $<23 \mathrm{~mm}$. Nevertheless, the method of measurement described by Kim et al. is rather complex; three lines and two angulations need to be set to obtain the measurement. ${ }^{4}$ In addition, this method of measurement has not been submitted to inter-observer agreement studies.

The second problem pointed out by Cohen has to do with the anteroposterior alignment of the orifice of the right lumen of the R-DLT in regard of the right upper lobe bronchus (RULB) opening. To overcome this, we proposed an R-DLT modification that consists of an enlarged lateral orifice area that allows a better positioning of the R-DLT. ${ }^{5}$ At that time, an accurate measurement of the RULB ostium angulation was not available, and our modification of the R-DLT was guided by clinical intuition only.

We wished to revisit the anatomy of the RMSB and that of the right upper lobe orifice to inform clinical practice in the operating room. More specifically, our primary objectives were: 1) to describe a novel and alternative method for measuring RMSB length, 2) to correlate its results with those obtained from Kim's method, and 3) to show the variability in angulation of the ostium of the RULB. A secondary objective was to evaluate the interobserver reproducibility of each of the methods of measure that we used.

\section{Methods}

A prospective observational cohort study was conducted. The Comité d'éthique de la recherche de l'Institut universitaire de cardiologie et de pneumologie de Québec evaluated the protocol on 19 April 2018 and granted a waiver of research ethics approval. 
Fig. 1 Measure of the right main stem bronchus length. The length of the right main stem bronchus (RMSB) was measured on the coronal plane in the axis of the RMSB for the two methods of measurement: Kim's method (Fig. 1A) and carina-to-carina measure (Fig. 1B). See text for details
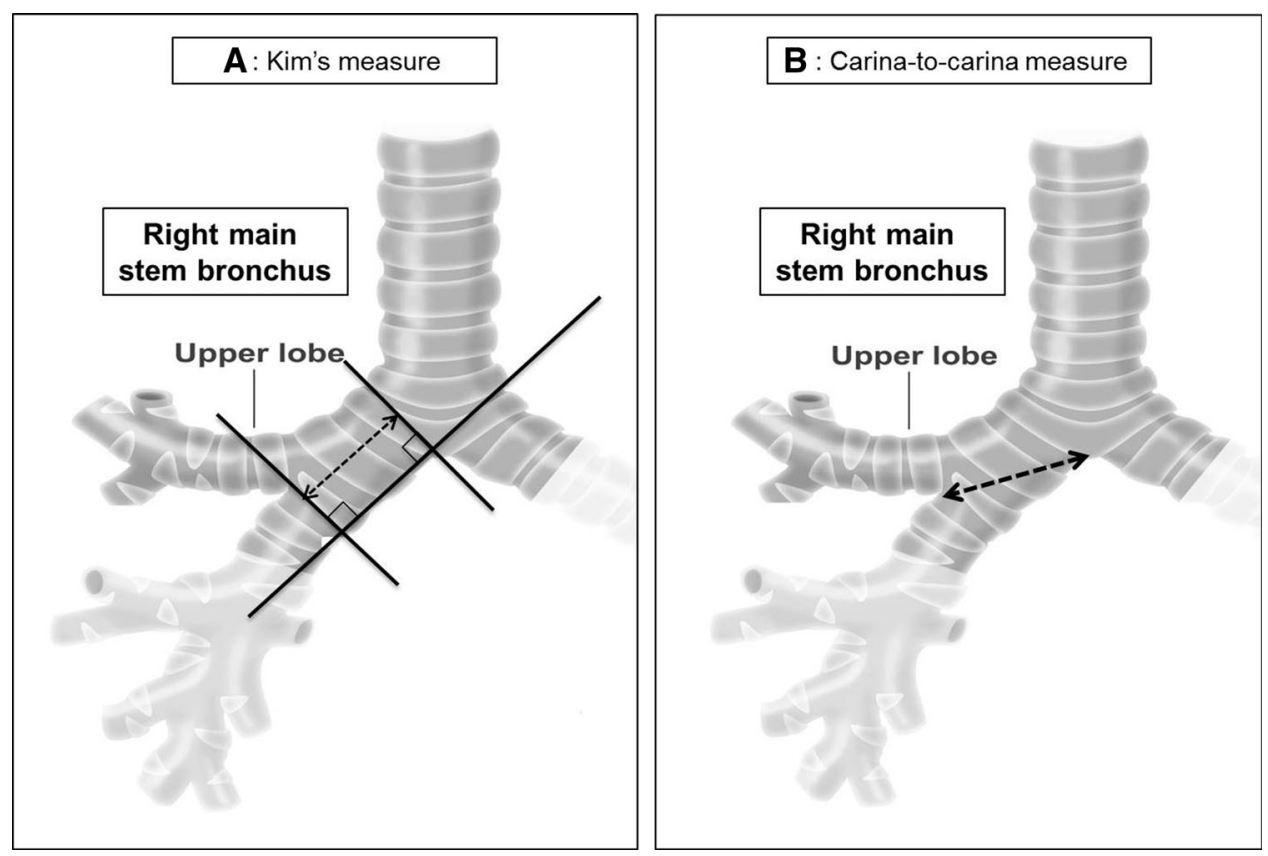

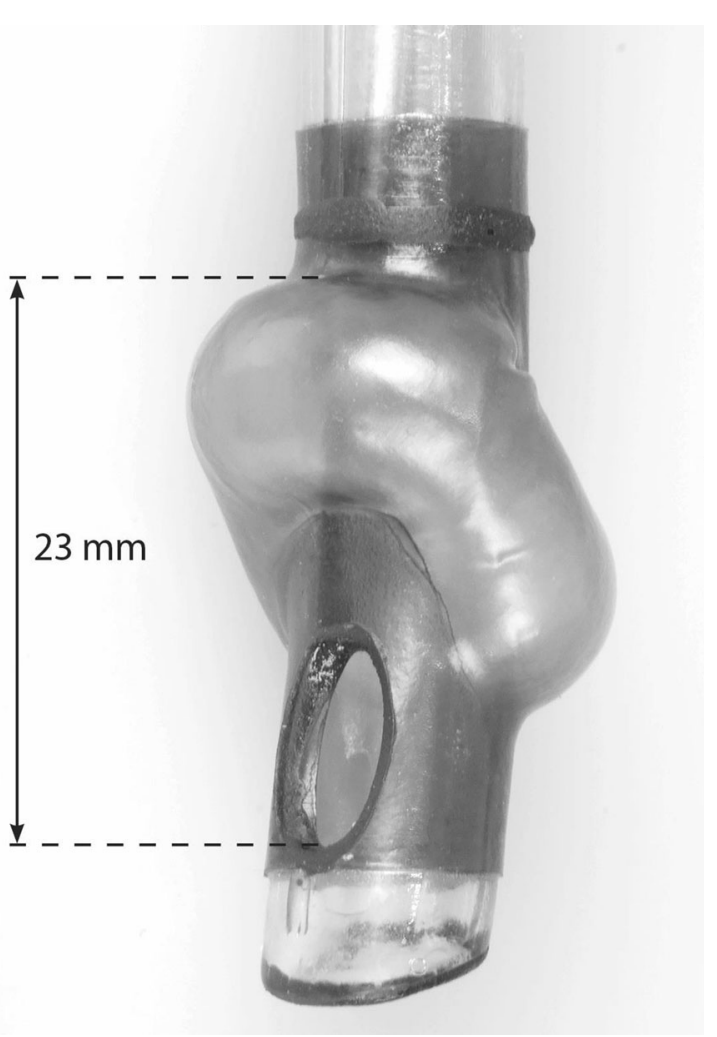

Fig. 2 Right side double lumen tube. The Kim's measure $(23 \mathrm{~mm})$ corresponds to the distance from the proximal end of the endobronchial cuff to the distal edge of the ventilation slot of the Broncho-Cath double lumen tube (Mallinckdrot Medical Ltd, Athlone, Ireland)
Population

Consecutive patients submitted to CT scan imaging of the thorax for diagnostic or follow-up purposes were included during a three-month period. No CT scan was done specifically for this study. Only patients with mature trachea and bronchi were included. A previous study showed that tracheobronchial tree growth is not completed before the age of 30 in women and 35 in men. Accordingly, only patients aged between 35 and $85 \mathrm{yr}$ old were included. ${ }^{6}$ As the intent was to describe normal anatomy, patients with tracheobronchial tree pathologies (e.g., tracheomalacia, tracheobronchomegaly, endobronchial lesions, bronchiectasis), mediastinal pathologies with extrinsic compression on the tracheobronchial tree, pulmonary pathologies inducing a distortion of the tracheobronchial tree (e.g., atelectasis, pulmonary fibrosis), prior lung resection, musculoskeletal deformity, and low-quality CT scan exams (e.g., significant kinetic artifacts in slices where measurements should be taken) were excluded.

Right upper lobe anatomy determination

Standard contrast CT scan images of the thorax were obtained from a 16-detector helical CT scanner (Seimens Somatom Definition, Siemens Healthcare Headquarters, Erlangen, Germany) that was operated using the following protocol: $5.0 \mathrm{~mm}$ slice thickness, 1.5 pitch, $120 \mathrm{kV}, 240$ 
Fig. 3 Measure of the angulation of the right upper lobe bronchus. Angle between a horizontal line passing by the centre of the right main stem bronchus lumen and a second line passing in the middle of the right upper lobe bronchus ostium

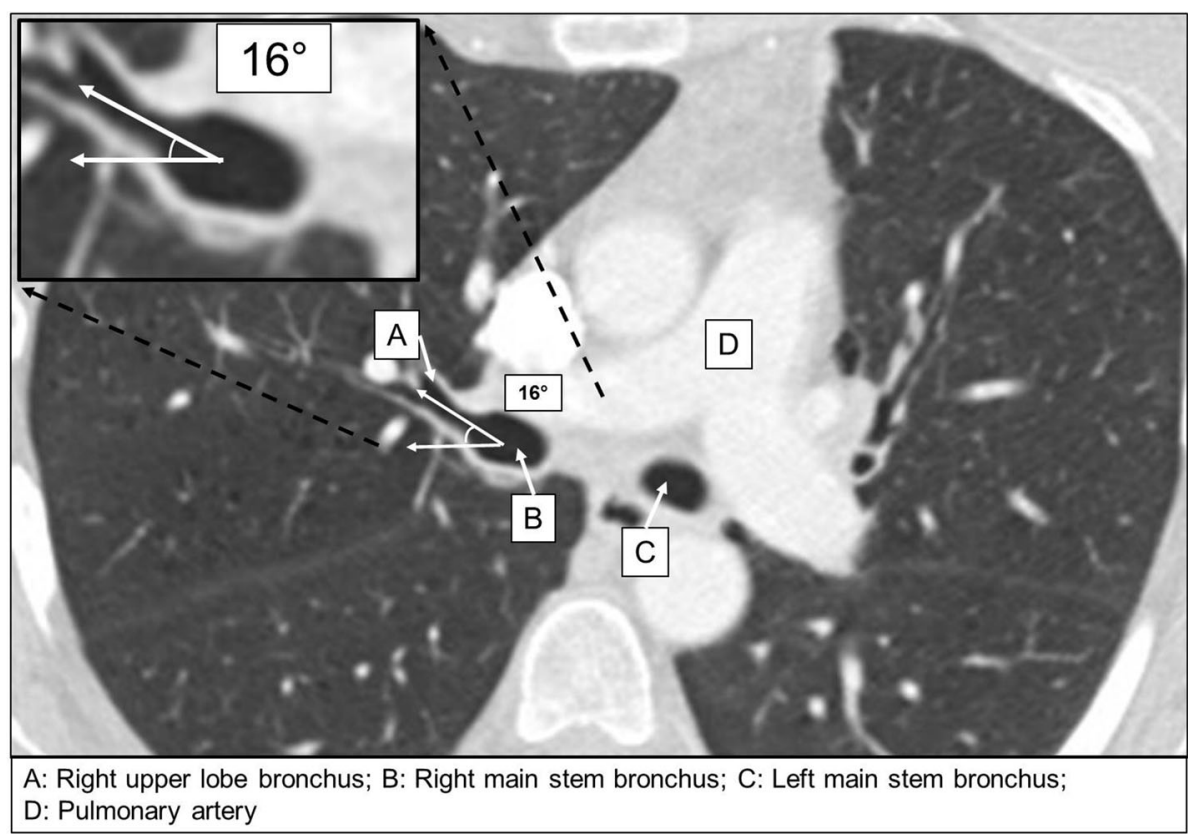

$\mathrm{mA}, \quad 0.8$ second tube rotation. The radiologic measurements were taken by a radiologist (M.G.) and a fellow in radiology (L.P.) who independently interpreted the $\mathrm{CT}$ scans before reaching consensus.

Right main stem bronchus length

We compared the measures of RMSB obtained from Kim's method (Fig. 1A) with those of an alternative method that we called the "carina-to-carina method" (Fig. 1B). ${ }^{4}$ For both methods, RMSB measures were taken on the coronal plane in the axis of the RMSB, on the frame that showed a clear tracheal carina and the largest diameter of the RMSB. With Kim's method (Fig. 1A), after drawing a line from the distal margin of the right upper lobe orifice, crossing perpendicular to the lower side of the right main bronchus, the distance between the carina and the crossing point was measured. With the carina-to-carina method (Fig. 1B), the distance between the tracheal carina and the first right interlobar carina (which separates the superior right upper lobe and the intermediate bronchus) was measured. It is noteworthy that the measure obtained from the carina-tocarina method does not exactly represent the length of the RMSB, but rather corresponds to the hypotenuse of a rightangled triangle whose adjacent side is the length of the RMSB, as measured with Kim's method.

Right upper lobe bronchus angulation

We measured the anteroposterior angulation between the RULB and the RMSB from a CT two-dimensional reconstruction in the plane of the bronchus from sagittal view, on the slice showing the widest opening of the RULB ostium (Fig. 3). Two lines were drawn: 1) a first horizontal line passing by the centre of the RMSB lumen; 2) a second line passing in the middle of the RULB ostium and joining the first line. The angulation was measured at the intersection of these two lines.

\section{Statistical analysis}

Proportions, means, standard deviations (SD), and ranges, when appropriate, were used to describe the study population. For clarity, we report only the mean values of all measurements (RMSB length and the RULB angulation) obtained by the two observors. We examined the correlation between the measures of the RMSB obtained from the two methods using the intraclass correlation coefficient (ICC). In both the RMSB length and the RULB angulation analyses, we reported the agreement between the two independent observers by computing ICCs. The ICCs obtained from the measurement made from Kim's and the carina-to-carina methods were also compared. In all analyses, statistical significance was set at 0.05 .

\section{Results}

Population

Two hundred consecutive radiologic thoracic CT scans done for diagnostic or follow-up purposes in as many patients were screened. Ninety-four were excluded for the 
following reasons: pulmonary resection $(n=36)$; lung cancer $(n=18)$; bronchiectasis $(n=10)$; pulmonary fibrosis $(n=8)$; mediastinal mass $(n=8)$; pleural effusion $(n=5)$; pneumonia $(n=2)$; cardiac surgery $(n=2)$; patients older than $85 \mathrm{yr}$ or younger than $35 \mathrm{yr}(n=1)$; other $(n=8)$. Data from one hundred and six patients [54 women; mean (SD) age: 62 (11) yr; height: 167 (10) cm; weight: 75 (17) kg] contributed to this analysis.

Right main stem bronchus length

From the Kim's method, the mean (SD) length of the RMSB was 25.5 (4.7) $\mathrm{mm}$ (range: 13.5-39.0 mm). The distribution of measurements using this latter method is shown in Fig. 4. Twenty-five percent of our patients had a RMSB $<23 \mathrm{~mm}$, a measure that predicts R-DLT malpositioning according to Kim's study. ${ }^{4}$ From the alternative carina-to-carina method, the mean (SD) length of the RMSB was 29.4 (4.6) $\mathrm{mm}$ (range: 19.1-40.8 mm). Overall, the inter-observer agreement was substantial with both methods (carina-to-carina method: $\mathrm{ICC}=0.95$; Kim's method: ICC $=0.84)$; the difference was however significant $(P<0.001)$. Both measures were closely related (ICC $=0.93 ; P<0.001$; Fig. 5). From this regression, we determined that a measure of $<26.7 \mathrm{~mm}$ (95\% confidence interval, 23.1 to 30.2 ) obtained with the carina-to-carina method corresponds with Kim's method to a measure of $<23 \mathrm{~mm}$. The upper limit of the confidence interval may be seen as a conservative estimate since the longest RMSB we actually observed with the carina-tocarina method corresponding with Kim's method to $<23$ mm measured $27.9 \mathrm{~mm}$.

Right upper lobe bronchus angulation

The mean (SD) RUL angle was $0.1(9.5)^{\circ}$. The angulation ranged from $21.2^{\circ}$ in the anterior direction to $-28.6^{\circ}$ in the posterior direction (Fig. 6). In the inter-observer agreement analysis, the ICC was 0.89 .

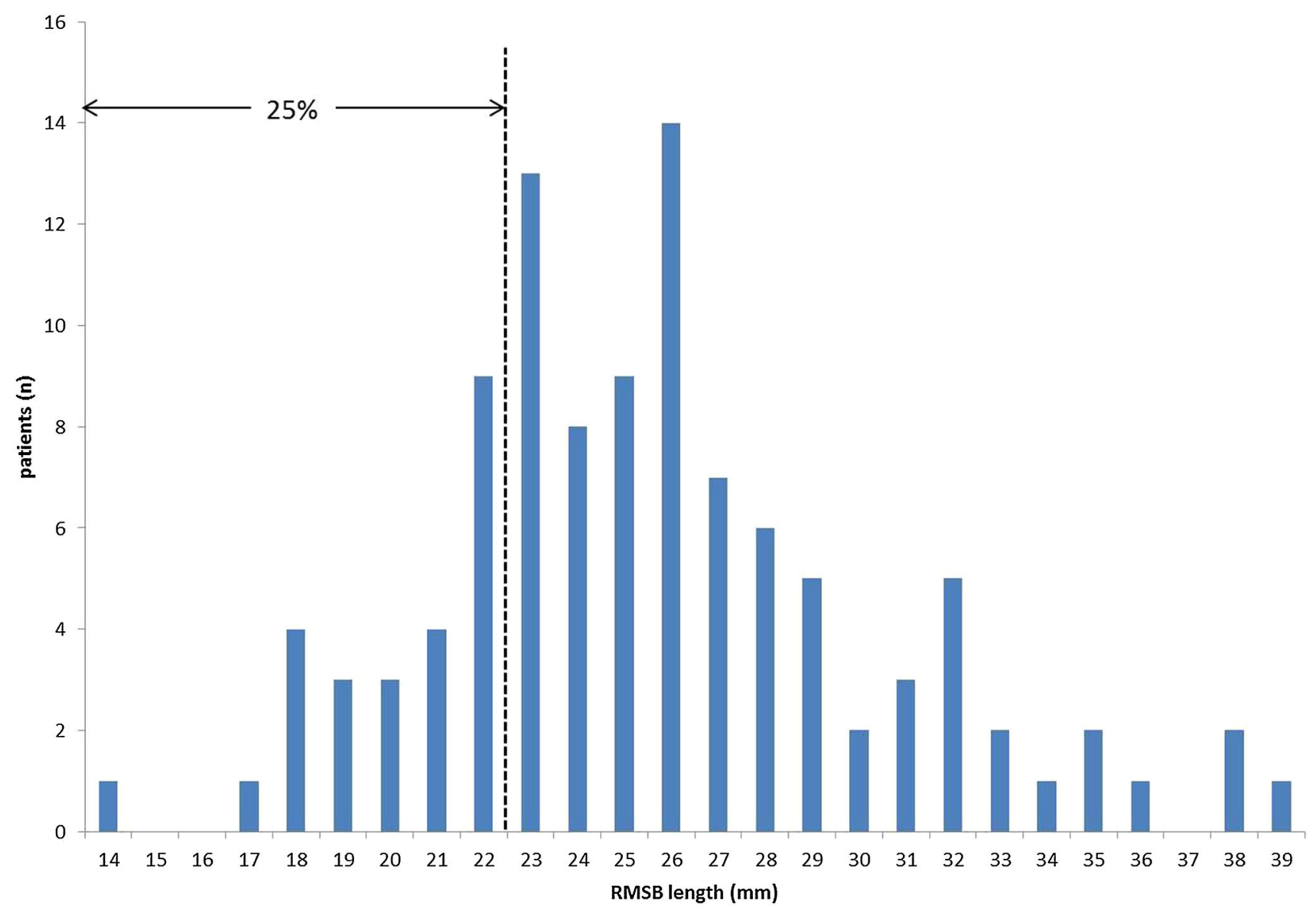

Fig. 4 Distribution of the right main stem bronchus length by Kim's method. Measurement of the distance between the tracheal carina and the distal margin of the right upper lobe bronchus. The length of the right main stem bronchus is less than $23 \mathrm{~mm}$ in $25 \%$ of cases 
Fig. 5 Measurement of the right main stem bronchus: correlation between Kim's and carina-to-carina methods. From linear regression analysis, a measure $<26.7 \mathrm{~mm}(95 \%$ confidence interval, 23.1 to 30.2) obtained with the carinato-carina method corresponds with a measure of $<23 \mathrm{~mm}$ obtained with Kim's method. This predicts right-sided double lumen tube malpositioning

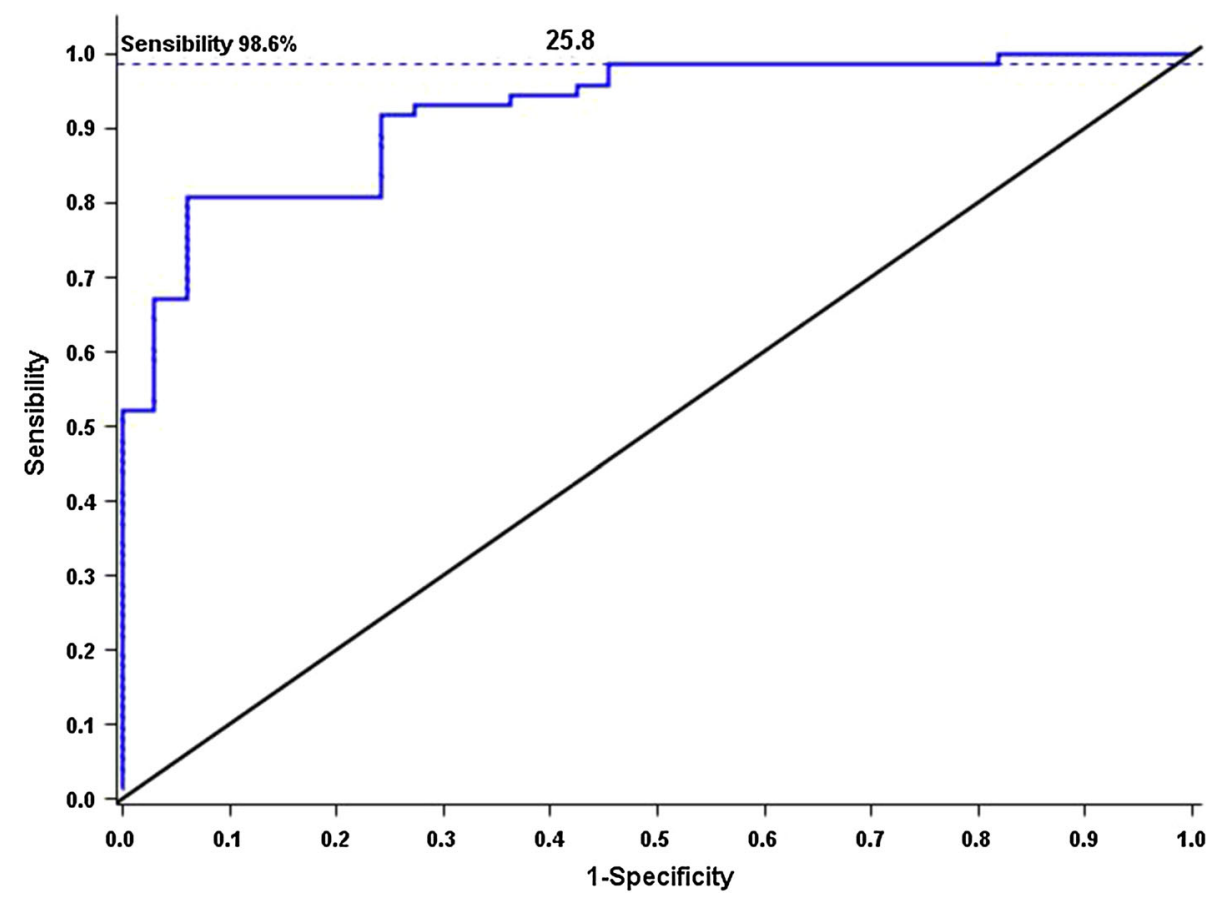

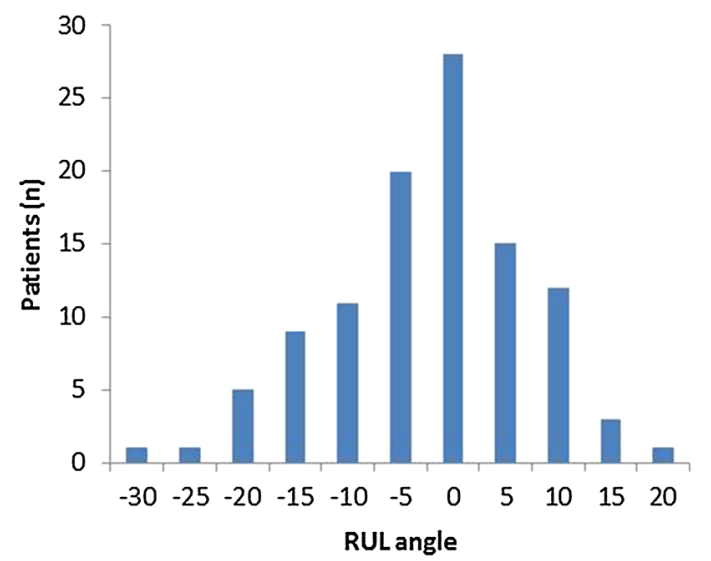

Fig. 6 Distribution of the right upper lobe bronchus angulation from the right main stem bronchus. Negative values are from posteriorly oriented right upper lobe bronchus (RULB) and positive values are from anteriorly oriented RULB

\section{Discussion}

The results of the first part of this investigation show an alternative and a highly reproducible method for measuring RSMB length. The measures obtained from this novel method correlate with those obtained from Kim's method. Nevertheless, the method we propose only requires one measure, whereas in the method previously described by Kim, three lines and two angulations need to be set to measure RSMB length. ${ }^{4}$ We submit that our alternative method of RMSB measurement could be easily learned by thoracic anesthesiologists after a short training and should be useful in choosing the lung isolation technique. Also, according to Kim's method, $25 \%$ of our population has a RMSB $<23 \mathrm{~mm}$, which may alert anesthesiologists regarding the possibility of R-DLT malpositioning.

The second part of this report confirms the hypothesis that the angulation between the origin of the RUL bronchus and the lateral aspect of the RMSB is highly variable. In our study, it ranged from $+21^{\circ}$ to $-29^{\circ}$ in the anteroposterior plane. This may at least in part explain the improvement in positioning obtained from the modified R-DLT with an enlargement of the lateral orifice that we reported in $2007 .{ }^{5}$ In this previous study, we showed that the success of initial positioning of the R-DLT in the supine position was similar with both standard and modified R-DLT. Nevertheless, immediately after turning patients to the lateral decubitus position, misalignment between the lateral orifice of the R-DLT and the origin of the RULB was more frequent with the standard version of R-DLT $(24 / 38,63 \%)$ than with the modified one $(10 / 38$, $26 \% ; P=0.012)$.

One limitation of our study is that the threshold of $<23$ $\mathrm{mm}$ that we use as the reference measure to predict R-DLT malpositioning arises from the specifications of the Broncho-Cath DLT only. Therefore, these results may not be applicable with other types of DLT's. Nevertheless, the Broncho-Cath DLT represents the majority of the RDLT used in North America. The strengths of our study are its fairly large sample size and that observations were made by two independent observers. 


\section{Conclusion}

This new information on RSMB length and RULB opening angulation may allow anesthesiologists to better understand the relatively complex anatomy of the right bronchial tree and to choose the best lung isolation device. A length $<27.9 \mathrm{~mm}$ measured by the carina-to-carina method could be used in clinical practice to alert anesthesiologists to the possibility of R-DLT malpositioning. The large variability of the RULB opening angulation strongly supports the need for wider utilization of the modified R-DLT with an enlargement of the lateral orifice. ${ }^{5}$

Conflicts of interest None declared.

Editorial responsibility This submission was handled by Dr. Gregory L. Bryson, Deputy Editor-in-Chief, Canadian Journal of Anesthesia.

Author contributions Jean S. Bussières was involved in substantial contributions to study conception and design, data analysis and interpretation, drafting the article, and revising it critically for important intellectual content. Michel Gingras and Lindsay Perron were involved in substantial contributions to study conception and design, acquisition of data, interpretation of data, drafting the article, and revising the article critically for important intellectual content. Marili Frenette was involved in substantial contributions to study conception and design, data analysis and interpretation, and revising the article critically for important intellectual content. Etienne J. Couture and Olivier Moreault were involved in substantial contributions to study conception and design and revising the article critically for important intellectual content. Jacques Somma and Yves Lacasse were involved in substantial contributions to study conception and design, interpretation of data, drafting the article, and revising the article critically for important intellectual content.

Funding Anesthesiology research team, Department of Anesthesiology, Institut Universitaire de Cardiologie et de Pneumologie de Québec - Université Laval.

\section{References}

1. Benumof JL, Partridge BL, Salvatierra C, Keating J. Margin of safety in positioning modern double-lumen endotracheal tubes. Anesthesiology 1987; 67: 729-38.

2. Cohen E. Methods of lung separation. Curr Opin Anaesthesiol 2002; 15: 69-78.

3. Pedoto A. How to choose the double-lumen tube size and side: the eternal debate. Anesthesiol Clin 2012; 30: 671-81.

4. Kim JH, Park SH, Han SH, Nahm FS, Jung CK, Kim KM. The distance between the carina and the distal margin of the right upper lobe orifice measured by computerised tomography as a guide to right-sided double-lumen endobronchial tube use. Anaesthesia 2013; 68: 700-5.

5. Bussieres JS, Lacasse $Y$, Cote D, et al. Modified right-sided Broncho-Cath double lumen tube improves endobronchial positioning: a randomized study. Can J Anesth 2007; 54: 276-82.

6. Jit H, Jit I. Dimensions \& shape of the trachea in the neonates, children \& adults in northwest India. Indian J Med Res 2000; 112: 27-33.

Publisher's Note Springer Nature remains neutral with regard to jurisdictional claims in published maps and institutional affiliations. 